\title{
Association Between Trauma Center Designation and Spinal Cord Injury Admission in the USA
}

\author{
Ross-Jordon S. Elliott ${ }^{\mathrm{a}, \mathrm{d}}$, Anand Dharia ${ }^{\mathrm{b}}$, Ali Seific
}

\begin{abstract}
Background: After spinal cord injury (SCI), patients are seen in either trauma center emergency departments (EDs) or non-trauma center EDs, and then selectively admitted for hospitalization. The association between SCI and admission to designated trauma centers is currently unknown. In this study, we assess the trends in admission between designated trauma centers after SCI from a large multi-center nationwide registry.
\end{abstract}

Methods: In this retrospective analysis of the Nationwide Emergency Department Sample (NEDS), we identified visits with SCI from 2006 to 2014. Z-test analyses were used to compare patients diagnosed with SCI at EDs with an associated trauma center designated hospital (TC-visits) against patients diagnosed with SCI at EDs without an associated trauma center designated hospital (NTC-visits).

Results: A total of 516,716 reported visits were identified with SCI. The annual total ED visits with admission to the same hospital for patients diagnosed with SCI increased significantly from 39,129 to $50,127$ from 2006 to 2014 ( $\mathrm{P}<0.001)$. From 2006 to 2014, the annual $\mathrm{ED}$ visits and admissions from TC-visits increased significantly from 27,781 to 43,926 and 23,445 to 35,635 , respectively $(\mathrm{P}<0.0001, \mathrm{P}<$ $0.0001)$. However, the annual ED visits and admissions from NTCvisits did not change significantly from 23,938 to 22,107 and 15,683 to 14,493 , respectively $(\mathrm{P}=0.09$ and $\mathrm{P}=0.1)$. Throughout the entire study period, the annual total $\mathrm{ED}$ visits with admissions to the same hospital was significantly higher for TC-visits than NTC-visits diagnosed with SCI $(\mathrm{P}<0.0001)$. The mean length of stay $(14.1$ days vs. 8.1 days), annual total in-hospital mortality (6.8\% vs. $6.0 \%)$, and annual total discharges to another institution (53.8\% vs. $46.8 \%)$ were significantly higher in TC-visits throughout the study period (P

Manuscript submitted June 30, 2020, accepted August 8, 2020

Published online September 4, 2020

aDepartment of Neurological Surgery, The George Washington University Hospital, Washington, DC 20037, USA

bDepartment of Neurological Surgery, Kansas University Medical Center, Kansas City, Missouri, USA

'Department of Neurological Surgery, University of Texas Health Science Center, San Antonio, Texas, USA

${ }^{\mathrm{d} C o r r e s p o n d i n g ~ A u t h o r: ~ R o s s-J o r d o n ~ S . ~ E l l i o t t, ~ D e p a r t m e n t ~ o f ~ N e u r o l o g i-~}$ cal Surgery, The George Washington University Hospital, 900 23rd St. NW, Washington, DC 20037, USA. Email: Relliott1@gwu.edu

doi: https://doi.org/10.14740/jnr609
$<0.001)$. However, the annual total routine discharges $(27.2 \%$ vs. $26.4 \%)$, annual total discharges to short-term hospital $(12.4 \%$ vs. $7.2 \%)$, and annual total discharges to home health care $(7.7 \%$ vs. $4.4 \%$ ) were significantly higher in NTC-visits throughout the study period $(\mathrm{P}<0.001)$.

Conclusions: Of the population of patients with SCI who visit EDs, those seen at trauma centers have a significant parallel association with incidence and patient outcome compared against those seen at non-trauma centers. Prospective research is warranted to make recommendations for required healthcare infrastructures based on an institution's trauma center designation.

Keywords: Spinal cord injury; Trauma; Trauma center; Mortality; Hospital length of stay; Disposition

\section{Introduction}

In the USA, there are an estimated 12,500 cases of spinal cord injury (SCI) that are newly diagnosed annually [1]. SCI can result in morbid disruption of spinal cord integrity, which can negatively impact a patient's quality of life, and occasionally lead to permanent disability [2]. In the acute injury period, clinical studies are equivocal on the efficacy of various treatment paradigms on patient outcomes in the severely injured patient. However, in patients with sub-total SCI, injury with incomplete motor and/or sensory losses, early intervention and rehabilitation have demonstrated robust improvements in morbidity $[3,4]$. To this end, primary trauma centers have the advantage of rapidly available multidisciplinary care teams, best suited for the care of this population. Data have suggested that primary management of traumatic SCI at level 1 trauma facilities may result in improved outcomes and shorter hospitalizations [5].

In the USA, the American College of Surgeons validates a trauma center's designation, which indicates the level of acute care that the institution can provide [6]. This designation identifies and categorizes emergency departments (EDs) and their associated hospitals as trauma centers based on their ability to staff and provide care to patients affected by a wide range of traumatic injuries [6]. Conversely, an ED and its associated hospital without trauma center designation are defined as a non-trauma center, which does not have the resources or staff to manage patients with traumatic injuries. This tiered tri- 
Table 1. Annual ED Visits and Admissions After SCl at Trauma Center (TC) and Non-Trauma Center (NTC) in the USA From 2006 to 2014

\begin{tabular}{|llllllllll}
\hline & $\mathbf{2 0 0 6}$ & $\mathbf{2 0 0 7}$ & $\mathbf{2 0 0 8}$ & $\mathbf{2 0 0 9}$ & $\mathbf{2 0 1 0}$ & $\mathbf{2 0 1 1}$ & $\mathbf{2 0 1 2}$ & $\mathbf{2 0 1 3}$ & $\mathbf{2 0 1 4}$ \\
\hline Total annual ED visits after SCI & 51,719 & 52,479 & 56,516 & 56,692 & 59,014 & 59,059 & 58,261 & 56,947 & 66,029 \\
Total annual admissions to same & 39,129 & 39,285 & 42,721 & 41,837 & 45,505 & 44,857 & 43,586 & 41,965 & 50,127 \\
hospital from ED visits after SCI & $(75.67 \%)$ & $(74.86 \%)$ & $(75.59 \%)$ & $(73.78 \%)$ & $(77.11 \%)$ & $(75.95 \%)$ & $(74.81 \%)$ & $(73.69 \%)$ & $(75.92 \%)$ \\
TC visits after SCI & 27,781 & 29,439 & 31,522 & 30,438 & 33,268 & 46,774 & 43,984 & 38,897 & 43,926 \\
TC admissions after SCI & 23,445 & 23,848 & 25,701 & 24,811 & 28,120 & 36,678 & 33,739 & 29,839 & 35,635 \\
& $(84.39 \%)$ & $(81.01 \%)$ & $(81.53 \%)$ & $(81.51 \%)$ & $(84.53 \%)$ & $(78.42 \%)$ & $(76.71 \%)$ & $(76.71 \%)$ & $(81.12 \%)$ \\
NTC visits after SCI & 23,938 & 23,040 & 24,994 & 26,254 & 25,747 & 12,285 & 14,277 & 18,051 & 22,104 \\
NTC admissions after SCI & 15,683 & 15,437 & 17,020 & 17,026 & 17,385 & 8,179 & 9,847 & 12,126 & 14,493 \\
& $(65.5 \%)$ & $(67 \%)$ & $(68.1 \%)$ & $(64.85 \%)$ & $(67.52 \%)$ & $(66.58 \%)$ & $(68.97 \%)$ & $(67.18 \%)$ & $(65.57 \%)$ \\
P value for number of & $<0.0001$ & $<0.0001$ & $<0.0001$ & $<0.0001$ & $<0.0001$ & $<0.0001$ & $<0.0001$ & $<0.0001$ & $<0.0001$ \\
admissions after SCI & & & & & & & &
\end{tabular}

SCI: spinal cord injury; ED: emergency department.

age system enables greater resource concentration for patient populations requiring them and minimizes redundancy, while also systematically improving costs. However, recent reports demonstrate that socioeconomic and racial trends are associated with the severity and subsequent management of SCI [7, 8]. Additionally, small studies have identified that patients who were discharged home directly from the ED were more likely to present to a non-trauma center, suggesting the possibility of admission bias in these cases [9].

Considering the lack of data on the incidence of SCI admissions to designated trauma centers, we sought to determine the incidence and trend of SCI admissions to hospitals, and to assess the impact of designated trauma centers on this trend in the USA.

\section{Materials and Methods}

\section{Patient selection}

Using data from the Nationwide Emergency Department Sample (NEDS) we conducted a 9-year retrospective analysis of patients who were admitted with a primary diagnosis of SCI. Patient specific demographics, including age and gender, were assessed and compared. The NEDS represents the largest allpayer ED database in the USA. Our institution exempted this analysis from full review by the Institutional Review Board. Patients admitted between 2006 and 2014, with a primary diagnosis of SCI were identified by Clinical Classification Software Code 227, which included ICD-9 codes of 8064, 8065, 8068, 8069, 9072, 9522-9524, 9528, 9529, 34939, 80600-80639, 80660-80662, 80669-80672, 80679, and 95200-95219 [10].

\section{Methods and measurements}

Year-wise distribution of ED visits, hospital admissions from $\mathrm{ED}$, and percentage of visits admitted to hospital from ED for patients with SCI at ED with an associated trauma center designated hospital (TC-visits) and patients with SCI at ED without an associated trauma center designated hospital (NTCvisits) were described.

Year-wise distribution of incidence, mean length of stay (LOS), annual total in-hospital mortality, annual total routine discharges, annual total discharges to short-term hospital, annual total discharges to home health care, and annual total discharges to another institution for patients with SCI at TC-visits and NTC-visits were described.

\section{Statistical analysis}

The Z-test statistic was used to compare nominal groups. Parametric data were analyzed as mean \pm standard deviation (SD) with statistical significance defined as $\alpha<0.05$. Non-parametric data were represented as mean \pm interquartile range (IQR).

\section{Results}

From 2006 to 2014, a total of 516,716 reported visits were concordant with a diagnosis of SCI. Most cases (median $=41.5 \%$, $\mathrm{IQR}=40.5-43, \mathrm{P}<0.01)$ were between 18 to 44 years old, of which $74.8 \%$ were males. Overall, the annual total ED visits for all patients diagnosed with SCI in the ED increased significantly from 51,719 to 66,029 , with a mean of 57,412.2 ED visits $(\mathrm{SD}=4,161.4)$ occurring annually during the study period $(\mathrm{P}<$ 0.001). For all patients diagnosed with SCI, the annual total ED visits with admission to the same hospital increased significantly from 39,129 to 50,127 throughout the study period ( $\mathrm{P}<0.001)$. Throughout the study period, the total cohort did not change significantly with regards to the rate of visits per 100,000 (4.0 to $3.8, \mathrm{P}=0.05)$, the total mean $\operatorname{LOS}$ (13.6 days to 12.5 days, $\mathrm{P}=$ $0.14)$, or total mortality $(6.21 \%$ to $6.06 \%, \mathrm{P}=0.95)$.

From 2006 to 2014, the annual ED visits and admissions from TC-visits increased significantly from 27,781 to 43,926 and 23,445 to 35,635 , respectively (Table $1, \mathrm{P}<0.0001, \mathrm{P}<$ 
Table 2. Patient Outcomes After Spinal Cord Injury for Trauma Center (TC) and Non-Trauma Center (NTC) in the USA From 2006 to 2014

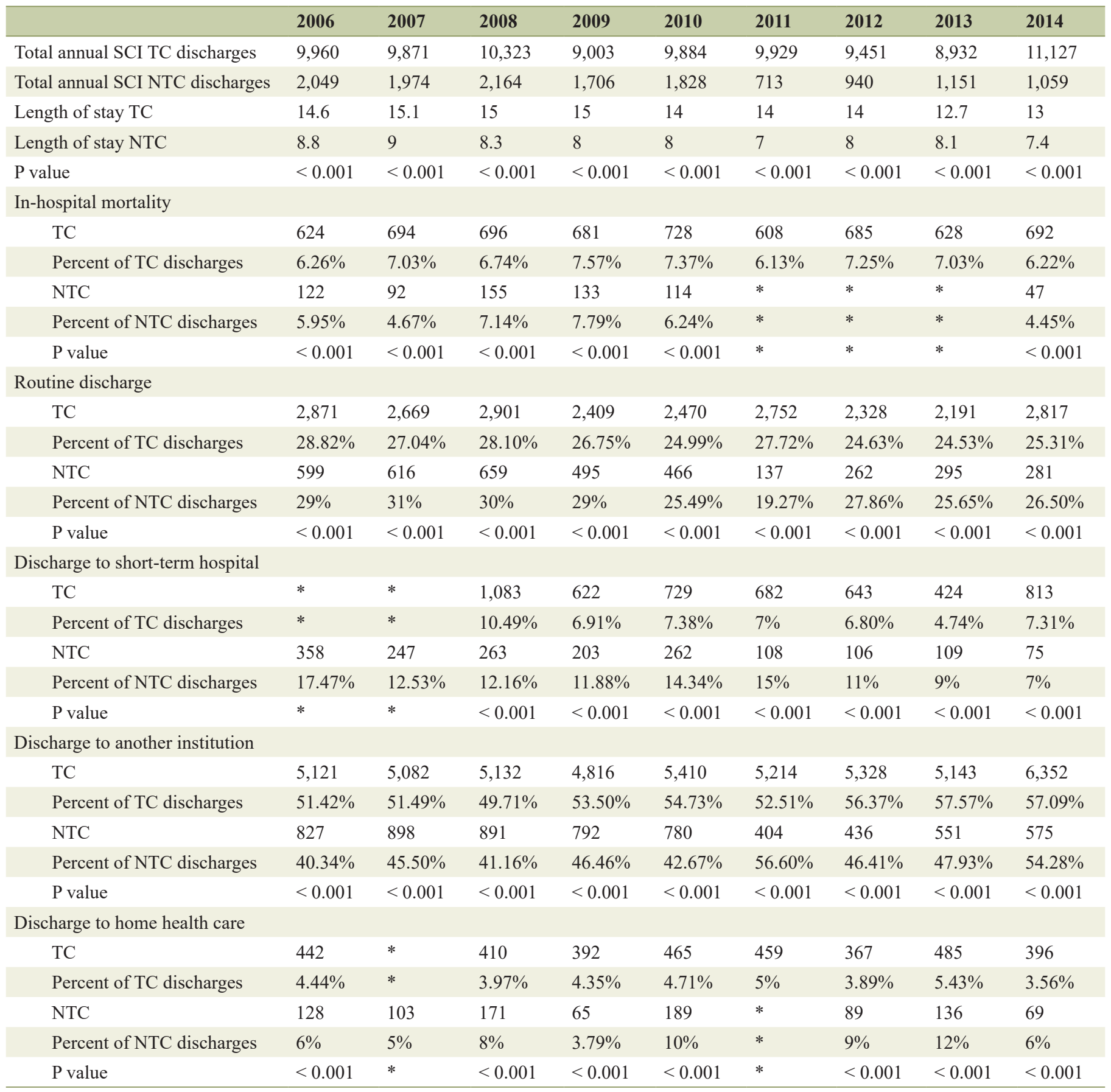

The numbers less than 10 are depicted with asterisks $\left({ }^{*}\right)$ for confidentiality based on the Health Care Utilization Project (HCUP) policy.

0.0001). However, the annual ED visits and admissions from NTC-visits did not change significantly from 23,938 to 22,107 and 15,683 to 14,493 , respectively (Table $1, \mathrm{P}=0.09$ and $\mathrm{P}$ $=0.1$ ). Throughout the entire study period, for patients with SCI, the annual total ED visits with same hospital admissions was significantly higher for TC-visits $(80.7 \%)$ than NTC-visits diagnosed with SCI (Table 1, P $<0.0001$ ).
The mean LOS (14.1 days vs. 8.1 days), annual total inhospital mortality $(6.8 \%$ vs. $6.0 \%)$, and annual total discharges to another institution (53.8\% vs. $46.8 \%)$ were significantly higher in TC-visits throughout the study period (Table 2, $\mathrm{P}<$ $0.001)$. However, the annual total routine discharges $(27.2 \%$ vs. $26.4 \%$ ), annual total discharges to short-term hospital $(12.4 \%$ vs. $7.2 \%)$, and annual total discharges to home health 
care $(7.7 \%$ vs. $4.4 \%)$ were significantly higher in NTC-visits throughout the study period (Table $2, \mathrm{P}<0.001$ ).

\section{Discussion}

In this study, we found a significant difference in the incidence of ED visits and hospital admissions for the population of patients suffering from SCI in trauma center designated hospitals (TC-visits) compared to non-trauma center hospitals (NTCvisits). Within the population of SCI, there was a consistently higher rate or incidence in TC-visits along with parallel associations in LOS, annual total in-hospital mortality, and annual total discharges to another institution. However, NTC-visits had significant parallel associations with annual total routine discharges, annual total discharges to short-term hospital, and annual total discharges to home health care. Overall, our results may highlight differences in the patient presentation, severity of initial injury, and/or inequalities in care between the two types of institutions.

The reason for these observed differences between EDs with and without trauma center designation may be multifactorial. It is important to acknowledge that in our own study, most patients with SCI were young adult males. This is in accordance with several previous studies that also determined young adult males to be a primary cohort in SCI [9-13]. These studies suggested that in this cohort, specifically, race and socioeconomic disparities further play an influential role in hospital admissions in the population of patients diagnosed with SCI [9-13]. These studies argued that patients in this demographic comprise patients with a lower socioeconomic status who may have limited access to primary care, which may further reduce ED utilization by this population [12]. On the contrary, the existing literature also suggests that the elderly cohort is associated with decreased admission rates following SCI even though elderly patients are more susceptible to SCI from relatively low mechanisms of injury $[14,15]$. These studies emphasize that patient demographics including socioeconomic status, race, age, and gender may more profoundly affect the incidence of patients diagnosed with and admitted for SCI than previously realized. While our study cannot directly compare and control for differences in the patient demographics between TC-visits and NTC-visits, it is nevertheless important to consider the impact of our own study's primary demographic in the context of our results.

In addition to patient demographic, differences in degree of injury severity likely accounts for differences in admissions and outcomes between the two institutions. Mohan et al demonstrated that physicians practicing in non-trauma centers encounter patients with moderate to severe injuries at a significantly lower rate than their trauma center physician counterparts [16]. This emphasizes the propensity for moderate-tosevere traumatic injuries to present to trauma centers. Lower SCI severity in patients seen at non-trauma centers could explain the lower mortality, shorter stays, and increased routine disposition observed in our own study. Future studies comparing the injury severity score (ISS), an established grading system for assessing traumatic severity, in these two institutions would therefore allow us to better comment on the impact of the trauma center's designation on patient outcomes. Moreover, follow-up metrics after discharge would allow us to better assess long-term outcomes in these SCI patients.

Lastly, inequalities in the level of care between TC and NTC-visits may exist. Patient signs and symptoms following SCI are highly variable, depending on the severity of injury and patient population $[3-5,14,15,17]$. Grassner et al reported rapidly progressing paresis $(49.5 \%)$ and abundance of respiratory insufficiency $(26.9 \%)$ as the most common reasons for neurological intensive care unit (ICU) admission following SCI in patients [17]. However, physiologic responses are not as easily detected in certain cohorts. For example, previous investigations have demonstrated that current trauma center triage filters may under-triage and thereby decrease hospital admission rates for elderly patients with SCI. Elderly patients, with impaired autoregulation and home B-blocker use, are likely to have normal heart rates following SCI, which is more likely to lead to fewer admissions in this cohort despite the degree of injury sustained $[14,15,18-23]$. It is possible, then, that EDs with trauma center designated hospitals, with their rapidly available multidisciplinary care teams, may detect SCI signs and symptoms more readily than their non-trauma center counterparts [3-5]. This would result in higher admission rates for patients visiting ED with trauma center designation, as was identified throughout our own study. Further investigation with regards to time to admission after insult may help to evaluate differences in care at these two types of institutions.

Information involving trauma center designation and its effect on hospital admissions and patient outcomes after SCI, including mortality, may play an influential role in the process by which SCI patients choose their care providers. While several studies argued in favor of the superiority of trauma centers, highlighting significantly fewer complication rates, lower mortality, and better overall patient outcomes than non-trauma centers, our study found patients treated at trauma centers experienced a higher mortality rate and a longer LOS [24, 25-30]. We propose that a larger proportion of severe cases of SCI presented to trauma centers, which may have influenced the higher mortality rate in trauma centers for this study. The number of TC-visits significantly increased during the study period, whereas the number of NTC-visits significantly decreased. This may suggest that improved outcomes and acknowledged care at trauma centers influenced patients' choice of care over the study period.

Although our study design allows for a large dataset over a wide range of care settings, our study has several limitations that may affect the interpretation of reported findings. The NEDS does not account for number of patients, but for visits to the ED. Some patients may have had multiple visits to the ED following SCI and may have therefore been accounted for multiple times. The NEDS utilizes large sample sizes and we acknowledge the need for validation in additional datasets. Additionally, presented data may include transfers of patients from non-trauma centers to trauma centers, which could have impacted our calculations. Finally, generalizability of our study to countries other than the USA may not be possible as healthcare settings may differ. Nevertheless, information involving trauma center designation and its effect on hospital admissions and patient outcomes after SCI has significant implications on the clinical care of SCI, the financial aspects of 
treatment of SCI, and our understanding of healthcare resources. Future studies comparing for SCI injury severity, patient demographic factors, complications at follow up, and time to admission could provide invaluable insight into differences in care between these two care settings, as described previously.

In summary, in this large nationwide study, the yearly observation of SCI patients consistently showed that the trauma center designation of hospitals associated with EDs paralleled associations in incidence and patient outcomes. Considering the severity of this clinical condition, the outcomes of patients with this pathology is significant for both patients and healthcare institutions. In the population studied, trauma centers had a significantly higher rate of SCI incidence and hospital admission from ED. Also, the mean LOS, annual total in-hospital mortality, and annual total discharges to another institution were significantly higher in trauma centers throughout the study period. Knowing this information may be beneficial for patients diagnosed with SCI to assist in plans in regards to SCI treatment. Prospective research is warranted to make recommendations for patient care following SCI based on the trauma center designation.

\section{Acknowledgments}

None to declare.

\section{Financial Disclosure}

There were no external sources of financial support for this manuscript.

\section{Conflict of Interest}

The authors report no conflict of interest concerning the materials or methods used in this study or the findings specified in this paper.

\section{Informed Consent}

Informed consent was not required by our institution for this study.

\section{Author Contributions}

RSE performed research including data collection, statistical analysis, created tables and wrote the paper. AD wrote the paper. AS designed research and provided expert clinical knowledge to revise critically.

\section{Data Availability}

The data supporting the findings of this study have been de- posited in HCUPnet database and can be accessed at https:// hcupnet.ahrq.gov/\#setup.

\section{References}

1. National Spinal Cord Injury Statistical Center (NSCISC). Spinal cord injury facts and figures at a glance. 2014. https://www.nscisc.uab.edu/PublicDocuments/fact_figures_docs/Facts\%202014.pdf. Accessed Mar 27, 2017.

2. Lee BA, Leiby BE, Marino RJ. Neurological and functional recovery after thoracic spinal cord injury. J Spinal Cord Med. 2016;39(1):67-76.

3. Wilson JR, LT BS, Aarabi B, et al. 181 Guidelines for the management of patients with spinal cord injury: the optimal timing of decompression. Neurosurgery. 2016;63(Suppl 1):172.

4. Furlan JC, Craven BC, Massicotte EM, Fehlings MG. Early versus delayed surgical decompression of spinal cord after traumatic cervical spinal cord injury: a costutility analysis. World Neurosurg. 2016;88:166-174.

5. Macias CA, Rosengart MR, Puyana JC, Linde-Zwirble WT, Smith W, Peitzman AB, Angus DC. The effects of trauma center care, admission volume, and surgical volume on paralysis after traumatic spinal cord injury. Ann Surg. 2009;249(1):10-17.

6. Society AT. Trauma center designation and verification by level of trauma care. 2015 .

7. Piatt JH, Jr. Pediatric spinal injury in the US: epidemiology and disparities. J Neurosurg Pediatr. 2015;16(4):463471.

8. Shin JI, Lee NJ, Cho SK. Pediatric Cervical Spine and Spinal Cord Injury: A National Database Study. Spine (Phila Pa 1976). 2016;41(4):283-292.

9. Selvarajah S, Schneider EB, Becker D, Sadowsky CL, Haider AH, Hammond ER. The epidemiology of childhood and adolescent traumatic spinal cord injury in the United States: 2007-2010. J Neurotrauma. 2014;31(18):1548-1560.

10. Cook AD, Ward JG, Chapple KM, Akinbiyi H, Garrett M, Moore FO, 3rd. Race and rehabilitation following spinal cord injury: equality of access for American Indians/ Alaska Natives compared to other racial groups. Inj Epidemiol. 2015;2(1):17.

11. Ametefe MK, Bankah PE, Yankey KP, Akoto H, Janney D, Dakurah TK. Spinal cord and spine trauma in a large teaching hospital in Ghana. Spinal Cord. 2016;54(12):1164-1168.

12. Guilcher SJ, Munce SE, Couris CM, Fung K, Craven BC, Verrier M, Jaglal SB. Health care utilization in nontraumatic and traumatic spinal cord injury: a populationbased study. Spinal Cord. 2010;48(1):45-50.

13. Yarbrough CK, Gamble PG, Burhan Janjua M, Tang M, Ghenbot R, Zhang AJ, Juknis N, et al. Readmission after spinal cord injury: analysis of an institutional cohort of 795 patients. J Neurosurg Sci. 2018;62(3):265-270.

14. Ma MH, MacKenzie EJ, Alcorta R, Kelen GD. Compliance with prehospital triage protocols for major trauma patients. J Trauma. 1999;46(1):168-175. 
15. Demetriades D, Sava J, Alo K, Newton E, Velmahos GC, Murray JA, Belzberg $\mathrm{H}$, et al. Old age as a criterion for trauma team activation. J Trauma. 2001;51(4):754-756; discussion 756-757.

16. Grassner L, Marschallinger J, Dunser MW, Novak HF, Zerbs A, Aigner L, Trinka E, et al. Nontraumatic spinal cord injury at the neurological intensive care unit: spectrum, causes of admission and predictors of mortality. Ther Adv Neurol Disord. 2016;9(2):85-94.

17. Furlan JC, Sakakibara BM, Miller WC, Krassioukov AV. Global incidence and prevalence of traumatic spinal cord injury. Can J Neurol Sci. 2013;40(4):456-464.

18. Scheetz LJ. Effectiveness of prehospital trauma triage guidelines for the identification of major trauma in elderly motor vehicle crash victims. J Emerg Nurs. 2003;29(2):109-115.

19. Scheetz LJ. Trauma center versus non-trauma center admissions in adult trauma victims by age and gender. Prehosp Emerg Care. 2004;8(3):268-272.

20. Zimmer-Gembeck MJ, Southard PA, Hedges JR, Mullins RJ, Rowland D, Stone JV, Trunkey DD. Triage in an established trauma system. J Trauma. 1995;39(5):922-928.

21. Phillips S, Rond PC, 3rd, Kelly SM, Swartz PD. The failure of triage criteria to identify geriatric patients with trauma: results from the Florida Trauma Triage Study. J Trauma. 1996;40(2):278-283.

22. Vassar MJ, Holcroft JJ, Knudson MM, Kizer KW. Fractures in access to and assessment of trauma systems. J Am Coll Surg. 2003;197(5):717-725.

23. Mohan D, Barnato AE, Rosengart MR, Farris C, Yealy DM, Switzer GE, Fischhoff B, et al. Trauma triage in the emergency departments of nontrauma centers: an analy- sis of individual physician caseload on triage patterns. J Trauma Acute Care Surg. 2013;74(6):1541-1547.

24. Smith JS, Jr., Martin LF, Young WW, Macioce DP. Do trauma centers improve outcome over non-trauma centers: the evaluation of regional trauma care using discharge abstract data and patient management categories. J Trauma. 1990;30(12):1533-1538.

25. Khuri SF, Daley J, Henderson W, Hur K, Hossain M, Soybel D, Kizer KW, et al. Relation of surgical volume to outcome in eight common operations: results from the VA National Surgical Quality Improvement Program. Ann Surg. 1999;230(3):414-429; discussion 429-432.

26. Kahn JM, Goss CH, Heagerty PJ, Kramer AA, O'Brien CR, Rubenfeld GD. Hospital volume and the outcomes of mechanical ventilation. N Engl J Med. 2006;355(1):41-50.

27. MacKenzie EJ, Rivara FP, Jurkovich GJ, Nathens AB, Frey KP, Egleston BL, Salkever DS, et al. A national evaluation of the effect of trauma-center care on mortality. N Engl J Med. 2006;354(4):366-378.

28. Mullins RJ, Mann NC, Hedges JR, Worrall W, Helfand M, Zechnich AD, Jurkovich GJ. Adequacy of hospital discharge status as a measure of outcome among injured patients. JAMA. 1998;279(21):1727-1731.

29. Meldon SW, Reilly M, Drew BL, Mancuso C, Fallon W, Jr. Trauma in the very elderly: a community-based study of outcomes at trauma and nontrauma centers. J Trauma. 2002;52(1):79-84.

30. Delgado MK, Yokell MA, Staudenmayer KL, Spain DA, Hernandez-Boussard T, Wang NE. Factors associated with the disposition of severely injured patients initially seen at non-trauma center emergency departments: disparities by insurance status. JAMA Surg. 2014;149(5):422-430. 